\title{
MEASURING YIELDS: ARITHMETIC, GEOMETRIC AND HORIZON-CONSISTENT AVERAGE
}

\section{Michal Dvořák*}

\begin{abstract}
:
The choice of averaging method has considerable impact on the average yield of a financial variable. Usually, geometric average is preferred, though dissenting opinions exist. Here it is shown that the problem has a consistent solution, which is called the horizon-consistent average. It is shown why geometric and arithmetic average calculations are almost always biased. When using company valuation's most common SP500 dataset by lbbotson Associates for 1928-2012 and the recommended 10-year forecasting horizon, consistent with the 10-year government securities in a CAPM model, the arithmetic average is severely flawed. On the other hand, the geometric average for similar horizons does not deviate much from the horizon-consistent average.
\end{abstract}

Keywords: yield, historical yield, arithmetic average, geometric average, risk premium JEL Classification: G1, G32

\section{Introduction}

Using this journal as a reference, most analyses of financial instruments return concentrate on investigating short-and medium-term performance (Dariusz 2013; Kavker - Festic, 2011; Trešl - Blatná, 2007; Bemerew, 1999; Trešl, 1999). This does not mean that the long-term performance is of lesser importance or free of dispute. In this article, we focus on one unresolved aspect in longer-term performance evaluation and forecasting - the proper averaging method for yield measurement.

The choice of averaging method has potentially considerable effect on the value of the average yield of a financial instrument. The question is relevant in all situations where multi-period yield measurement, benchmarking or forecasting are conducted. This includes financial risk management, performance of mutual funds and estimation of cost of equity capital, to name a few. The impact of method choice is amplified when longer horizon is considered. As company valuation usually expects companies to live over a prolonged period of time, the problem is disproportionately significant there. For this reason, this problem will be exposed from valuation perspective.

Company valuation employs CAPM-style models to determine the cost of capital. Equity premium inside CAPM is defined as the difference between a yield of market index and a yield of a risk free instrument. To evaluate each of these, geometric average of past data is usually preferred, though dissenting opinions exist and the issue is not resolved satisfactorily (Mařík et al., 2011, p. 312). Damodaran (2013b) favours geometric average on the grounds of negative autocorrelation and forecasting horizon differences, using

* Michal Dvořák, Faculty of Finance and Accounting, University of Economics, Prague, Department of Monetary Theory and Policy, and Czech National Bank (michal@michaldvorak.eu).

This paper has been supported by the University of Economics, Prague, IGA Grant No. 5/2014

"Finanční a hospodářský cyklus". 
the conclusions by Indro, Lee (1997). Nevertheless, the conclusions lack sound theoretical backing.

In this article, we start with an intuitive view of yield measures and develop a general procedure that is in accord with this view. The procedure is later referred to as horizonconsistent average. The solution is inferred under the independence of yields in time, which eases the exposition. The procedure can be further modified to account for autocorrelated yields. Making it a benchmark, respective biases of arithmetic and geometric average are investigated.

As an application, the averages are demonstrated on company valuation's most frequently used dataset of SP500 by Ibbotson Associates for 1928-2012.

\section{How Much Do the Averages Differ?}

It is a well-known mathematical fact that for any given numbers, arithmetic average is greater than geometric average. ${ }^{1}$ There is a single exception; both averages are equal if and only if all averaged numbers are identical. The greater is the difference between the numbers, the greater is the difference between their averages. Differences between the numbers can be measured by their standard deviation. Because stock yields exhibit higher standard deviation (in time) than less risky government securities, the difference between averages is greater in the case of SP500 stock index than in the cases of Treasury Bonds and Treasury Bills. This is shown in Table 1.

Table 1 | Comparison of the Differences between Arithmetic and Geometric Averages for Variously Volatile US Instruments

\begin{tabular}{|c|c|c|c|c|}
\hline & $\begin{array}{c}\text { Volatility } \\
\text { (standard deviation) }\end{array}$ & $\begin{array}{c}\text { Arithmetic } \\
\text { average }\end{array}$ & $\begin{array}{c}\text { Geometric } \\
\text { average }\end{array}$ & $\begin{array}{c}\text { Difference } \\
\text { between averages }\end{array}$ \\
\hline SP500 stock index & $19.89 \%$ & $11.26 \%$ & $9.31 \%$ & 1.95 p.p. \\
\hline 10-year T-Bonds & $7.69 \%$ & $5.38 \%$ & $5.11 \%$ & 0.27 p. p. \\
\hline 3-month T-Bills & $3.03 \%$ & $3.61 \%$ & $3.57 \%$ & 0.04 p. p. \\
\hline
\end{tabular}

Source: Damodaran (2013). Annual data for 1928-2012 period. Geometric average is defined by (33).

The difference between averages is rather small for government securities, but for stock index it is large enough to have practical consequences.

\section{The Setup}

The reason for averaging past data can be either backward looking need to describe past development or forward looking need to forecast future movement. Though everything stated in the text is valid for both cases ${ }^{2}$, we will discuss the issue from the forecasting

1 The proof for 2-number case is straightforward. General proof can be obtained by mathematical induction (Veselý, 2004, pp. 30-31). Cauchy's induction proof together with 4 other proofs is also available on the devoted Wikipedia website (http://en.wikipedia.org/wiki/Inequality_of_arithmetic_ and_geometric_means).

2 When looking backwards, $N$ should be called relevant period rather than forecasting horizon. 
perspective in order to be consistent with our valuation application. When estimating yields, two characteristics enter the analysis.

- Number of observations (denoted $T$ ). It refers to the size of the dataset of past data available. In our application to annual yields during the 1928-2012 period we have 85 observations $(T=85)$.

- Forecasting horizon (denoted $N$ ). It refers to the horizon for which the average yield is forecasted. When $N=1$, we forecast an average yield of a financial variable from now to 1 period on. When $N=2$, we predict an average yield of a financial variable from now to two periods on. Average yield is compound annual growth rate of the financial variable. ${ }^{3}$ From technical reasons, forecasting horizon should be in full multiples of the frequency of observation; when using annual observations, forecasting horizon should be in full years. ${ }^{4}$ In the text we assume annual observations.

As the number of observations is a feature of the dataset employed and forecasting horizon depends on the specific need of the user, these two parameters are independent of each other. Thus, $N=T, N<T$ and $N>T$ cases might well occur, though $N<T$ is probably the most common situation.

As we shall see later, the relationship between the forecasting horizon and the number of observation plays an important role when choosing the averaging method.

Figure 1 | Histogram of SP500 Annual Yields during 1928-2012

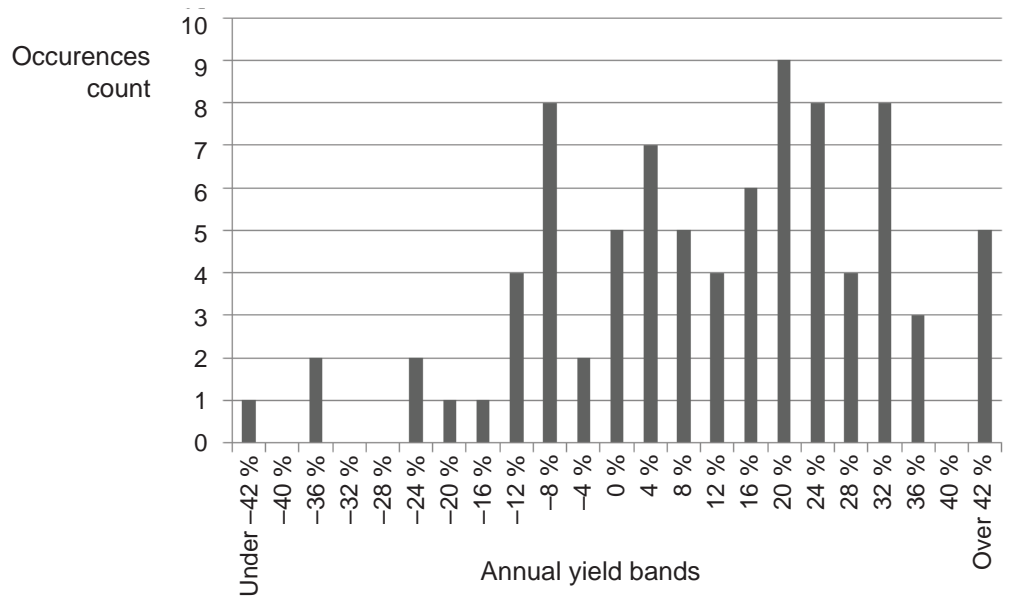

Source: Damodaran (2013). Yield bands reach 2\% both ways from the point stated on the horizontal axis, except for the leftmost and the rightmost band.

3 For 2-year horizon, it is a rate $g$, for which $I_{0} \cdot(1+g) \cdot(1+g)=I_{2}$, where $I_{0}$ is today's value of the financial variable and $I_{2}$ is its value after 2 years.

$4 \quad$ Procedures and conclusions of this article can be extended to horizons which are not full multiples of the frequency of observation. Nevertheless, considering such cases - for example, estimating weekly yield when using annual data or estimating a yield for 1.384 years - makes little practical sense. 
We begin with the distribution of historical yields of the financial variable. In our SP500 dataset by Ibbotson Associates (Damodaran, 2013), the resulting distribution's histogram is in Figure 1. As mentioned above, our objective is to predict future performance rather than to describe the past. The actual future performance is unknown, so we will consider it as a random variable, being probabilistically distributed according to the same distribution as occurred in the past (as in Figure 1). Thus, we employ past data to form an idea about probable yearly movement sizes and their likelihoods.

Two remarks should be made here. First, if all the past observations are relevant, larger number of observations delivers more proper description of the yields probability distribution. Oppositely, for example, with only 2 past data $(T=2)$, the distribution would be extremely rough and imprecise for future yield forecasting.

Second, the above was inferred under the suppositions that (1) all past yields employed in the distribution construction came from the same distribution and (2) that this yearly distribution remains unchanged for the forecasting period. If market structure has changed, yields realized under the old structure might be irrelevant for the new structure. This becomes an issue especially when trying to refine the yield distribution by extending the dataset to the past. Had stock market in 1928-1990 different yield-affecting characteristics from today's market, usage of old data can seriously impair forecasting accuracy instead of improving it.

In the following theoretical exposition is assumed all the data are relevant ${ }^{5}$. It accord with the prevailing valuation practice (Damodaran, 2013b), it is also assumed in our SP500 application.

One-period yield distribution. Being the basic building block of the analysis, the above-mentioned yield distribution demands formal treatment. Denote one-period yield $Y$. From modelling perspective, it is a random variable, distributed according to some distribution, called $D$. Information about distribution $D$ - its possible realizations and their respective probability of occurrence - can be derived from past data. For this purpose we can resort to the histogram in Figure 1. Nevertheless, the highest precision is obtained when all past observations are taken separately. This leads to the discrete probability distribution function of (1).

$$
D: Y=\begin{array}{cc}
x_{1} & \operatorname{Pr}\left\{x_{1}\right\}=t_{1} / T \\
x_{2} & \operatorname{Pr}\left\{x_{2}\right\}=t_{2} / T \\
\vdots & \vdots \\
x_{k} & \operatorname{Pr}\left\{x_{k}\right\}=t_{k} / T
\end{array}
$$

where $x_{i}$ denotes the $i$-th unique value of annual yield out of $k$ observed $t_{i}$-times in the dataset of $T$ values in total, leading to its respective probability of occurrence $\operatorname{Pr}\left\{x_{i}\right\}$. By the character of the setup it is very likely that almost all values will be recorded only once, leading to $t_{i}=1$ and $\operatorname{Pr}\left\{x_{i}\right\}=1 / T$.

Independence assumption. If more periods are considered (in the past or in future), more random variables describing yearly changes have to be considered. Label $Y_{1}$ random variable describing first period's yield, $Y_{2}$ random variable describing yield in the second period, until $Y_{T}$ is the random variable describing yield in the last considered period. In line

5 If this proves not to be realistic, two remedies can be made. Firstly, observations identified to come from different market structures can be skipped. Secondly, data can be weighted by relevance, with more relevant data receiving higher weight, interpreted as inflated number of occurrences. 
with the previous, suppose all these random variables share the same above-mentioned distribution $D$. It is then important to ask if they are independent of each other. Independence of distributions in time is empirically difficult to prove or disprove. Practitioners thus resort to testing yields autocorrelation. ${ }^{6}$ Although past studies (Fama, French, 1988) identified negative autocorrelation and some theorists work with autocorrelated yields, the 1928-2012 annual SP500 dataset exhibits autocorrelations very close to zero and statistically insignificant (see Table 2). Therefore, in our case, serial independence of yields assumption is very reasonable. This will substantially simplify the analysis.

Table 2 | Autocorrelation Coefficients for Annual Yields of SP500

\begin{tabular}{|l|c|c|c|}
\hline Lag & $\begin{array}{c}\text { Autocorrelation } \\
\text { coefficient (ACF) }\end{array}$ & $\begin{array}{c}\text { Autocorrelation coefficient adjusted } \\
\text { for interlaying lags (PACF) }\end{array}$ & $\begin{array}{c}\text { Ljung-Box Q statistics } \\
\text { [p-value] }\end{array}$ \\
\hline $\mathbf{1}$ & 0.0013 & 0.0013 & $0.0002[0.990]$ \\
\hline $\mathbf{2}$ & -0.1391 & -0.1391 & $1.7247[0.422]$ \\
\hline $\mathbf{3}$ & 0.0099 & 0.0105 & $1.7335[0.630]$ \\
\hline $\mathbf{4}$ & -0.0858 & -0.1073 & $2.4059[0.662]$ \\
\hline $\mathbf{5}$ & -0.0768 & -0.0756 & $2.9516[0.707]$ \\
\hline $\mathbf{6}$ & 0.0051 & -0.0240 & $2.9540[0.815]$ \\
\hline $\mathbf{7}$ & 0.1173 & 0.0996 & $4.2592[0.749]$ \\
\hline $\mathbf{8}$ & 0.0261 & 0.0165 & $4.3247[0.827]$ \\
\hline $\mathbf{9}$ & 0.0948 & 0.1183 & $5.1994[0.817]$ \\
\hline $\mathbf{1 0}$ & 0.0662 & 0.0697 & $5.6319[0.845]$ \\
\hline
\end{tabular}

Source: Damodaran (2013). Annual observations for 1928-2012 period.

\section{A Generally Inconsistent Arithmetic-Average Procedure}

Let us first present an appealing procedure which is justified when predicting on one horizon $(N=1)$ but turns out to be inconsistent if extended to multi-period forecasting $(N>1)$.

\subsection{Consistent forecast for one period $(N=1)$}

One-period forecasting is the following exercise. If the current value of SP500 is, for example, 1000, we are interested in the value of SP500 one year later. More precisely, we are interested in what is the yield given by such an index development. How to approach this problem? Assuming the yield distribution has not changed over time, it's logical to use a value which is "reasonable" in the light of past data. Statistically speaking, one-year yield $R_{1}$ is forecasted by the expected value ${ }^{7}$ of the annual yield distribution:

6 Independence implies autocorrelation, but not vice versa. If there is no autocorrelation, it might be due to independence, or there is a nonlinear relationship. When the independence assumption is based on zero autocorrelation, we hypothesize the first option is true.

7 The possibility to describe the distribution"s "center of gravity" differently, for example by the distribution's median, is left aside. 


$$
R_{1}=E\{Y\},
$$

where $E\{\}$ is the expectation operator.

The expected value of a random variable defined by (1) is

$$
E\{Y\}=\sum_{i=1}^{k}\left(x_{i} \cdot \operatorname{Pr}\left\{x_{i}\right\}\right)=\frac{1}{T} \cdot \sum_{i=1}^{T} x_{i} .
$$

Thus, it is equivalent to the arithmetic mean. For one-period forecasting, we used the arithmetic average of $T$ past values $x_{i}$ (later denoted $a_{A(T)}$ ) and not their geometric average (later denoted $a_{G(T)}$ ).

Association of the past data average with the distribution's mean is enabled by two facts. Firstly, expected value of a distribution is defined as a weighted arithmetic average (if all past data are unique, all weights are equal to $1 / T$ ). Secondly, the distribution is constituted by the very same values from which the arithmetic average is calculated. In Section 6.2 it is shown that geometric average does not meet the first fact, making such association impossible.

\subsection{Inconsistent forecast for more-than-one period $(N \geq 2)$}

The previous section's solution was very straightforward. More complicated situation occurs when trying to predict yield for more periods. When forecasting for $N$ periods, we ask, if today's value of SP500 is 1000 , what it will be after $N$ periods. More precisely, we examine the associated compound annual growth rate realized during this $N$-year period.

Intuitive solution can be best described in the following way. Consider this 8-step procedure.

1. Set the initial (today's) value of the financial variable to one, i.e. $I_{0}=1$.

2. Using the probability distribution of the original data $(D)$, randomly draw one value from this distribution and apply it to the index. If the randomly drawn value (a particular realization of $\left.Y_{1}\right)$ is called $y_{1}$, we obtain $I_{0} \cdot\left(1+y_{1}\right)=\left(1+y_{1}\right)=I_{1}$. We thus obtain the index value after one year $\left(I_{1}\right)$.

3. Draw another random number from $D$ distribution and chain-apply it on the index. If the drawn value (again a particular realization of $Y_{2}$ ) is $y_{2}$, we obtain $I_{0} \cdot\left(1+y_{1}\right) \cdot\left(1+y_{2}\right)=\left(1+y_{1}\right) \cdot\left(1+y_{2}\right)=I_{2}$. This is the index value after 2 years $\left(I_{2}\right)$.

4. Continue drawing independent random numbers and applying them to the index in the same manner until having reached the total number of $N$ independent draws. Then we obtain $I_{0} \cdot\left(1+y_{1}\right) \cdot\left(1+y_{2}\right) \cdot \ldots \cdot\left(1+y_{N}\right)=\left(1+y_{1}\right) \cdot\left(1+y_{2}\right) \cdot \ldots \cdot\left(1+y_{N}\right)=I_{N}$. We thus obtain the index value after $N$ years $\left(I_{N}\right)$.

5. Record the resulting index value after $N$ years - the number $I_{N}$. We label it $I_{N, 1}$ to explicitly mention it is the first of the many simulated final index values.

6. Repeat steps 2-5 many times or, ideally, pick all permissible combinations of draws. The number of such repetitions we call $j$.

7. Take the arithmetic average of the many resulting index values after years - numbers $I_{N, 1}, I_{N, 2}, \ldots, I_{N, j}$. We obtain the number of $\bar{I}_{N}=\frac{1}{j} \cdot \sum_{i=1}^{j} I_{N, i}$. 
8. Take the $N$-th root of the average index value after $N$ years to obtain compound annual growth rate of the index. In other words, annual yield, which delivers the average index value in $N$ years. This constitutes the $N$-year horizon forecast $\left(R_{N}\right): R_{N}=\sqrt[N]{\bar{I}_{N}}-1$.

Now we describe the process algebraically. Index value after $N$ years can be generally written as a product of $N$ random variables $Y_{i}$, all identically distributed with distribution $D$ (1) and mutually independent.

$$
I_{N}=1 \cdot\left(1+Y_{1}\right) \cdot\left(1+Y_{2}\right) \cdot \ldots \cdot\left(1+Y_{N}\right)
$$

The expected value (the arithmetic average) of index values after $N$ years will generally be

$$
E\left\{I_{N}\right\}=E\left\{\left(1+Y_{1}\right) \cdot\left(1+Y_{2}\right) \cdot\left(1+Y_{N}\right)\right\} .
$$

Because the expected value of the index after $N$ years can be calculated as the average of all possible combinations of one-year past yields $x_{i}$, the expression (5) can be sequentially simplified to the form of expression (9).

$$
\begin{gathered}
E\left\{I_{N}\right\}=\frac{1}{T^{N}} \cdot \sum_{i_{1}=1}^{T} \sum_{i_{2}=1}^{T} \ldots \sum_{i_{N}=1}^{T} \cdot\left[\left(1+x_{i_{1}}\right) \cdot\left(1+x_{i_{2}}\right) \cdot \ldots \cdot\left(1+x_{i_{N}}\right)\right] \\
E\left\{I_{N}\right\}=\frac{1}{T^{N-1}} \cdot \sum_{i_{2}=1}^{T} \ldots \sum_{i_{N}=1}^{T}\left[\left(1+x_{i_{2}}\right) \cdot \ldots \cdot\left(1+x_{i_{N}}\right) \cdot \frac{1}{T} \sum_{i_{1}=1}^{T}\left(1+x_{i_{1}}\right)\right] \\
E\left\{I_{N}\right\}=\left(1+E\left\{Y_{1}\right\}\right) \cdot\left(1+E\left\{Y_{2}\right\}\right) \cdot \ldots \cdot\left(1+E\left\{Y_{N}\right\}\right)=\left(1+R_{1}\right) \cdot \ldots \cdot\left(1+R_{1}\right) \\
E\left\{I_{N}\right\}=\left(1+R_{1}\right)^{N}
\end{gathered}
$$

See that to forecast expected value of the index after $N$ years, only the expected value of the 1 -year yield is needed. The annualized $N$-year forecast (see step 8 of the procedure) is

$$
\begin{gathered}
R_{N}=\left[E\left\{\left(1+Y_{1}\right) \cdot\left(1+Y_{2}\right) \cdot \ldots \cdot\left(1+Y_{N}\right)\right\}\right]^{1 / N}-1=\left[\left(1+R_{1}\right)^{N}\right]^{1 / N}-1 \\
R_{N}=R_{1} .
\end{gathered}
$$

As $N$ can be any natural number, the forecasts are identical for all horizons and are equal to the 1-year forecast. It looks like arithmetic, and not geometric, average is the right way to calculate average yields, irrespectively of the forecasting horizon $N$. This would counter the conclusions of Indro, Lee (1997) and recommendations in Damodaran (2013b).

Nevertheless, the procedure, specifically steps 7 and 8, contains a flaw. This is best seen when $N=85$. From Table 1 we know the arithmetic average of the SP500 data $\left(a_{A(85)}\right)$ to be $11.26 \%$. Setting the initial index value $I_{0}=1$, the terminal index value $\left(I_{85}\right)$ is:

$$
I_{85}=I_{0} \cdot\left(1+a_{A(85)}\right)^{85}=1.1126^{85}=8686 .
$$

When the initial value is sequentially adjusted for annual yields which materialized each year, being the way how the data were acquired, we reach the terminal value (see geometric average $a_{G(85)}=9,31 \%$ in Table 1$)$ 


$$
I_{85}=I_{0} \cdot\left(1+x_{1}\right) \cdot\left(1+x_{2}\right) \cdot \ldots \cdot\left(1+x_{85}\right)=\left(1+a_{G(85)}\right)^{85}=1.0931^{85}=1932 .
$$

This documents that arithmetic averaging, though apparently correct through equation (11), substantially overstates reality; in this case by the factor of almost 4.5! For exhibiting this odd behaviour, we call the procedure inconsistent for horizon 85 years. Moreover, this was the case of zero autocorrelation. Were negative autocorrelation in place, then

$$
E\left\{I_{N}\right\}<E\left\{\left(1+Y_{1}\right) \cdot\left(1+Y_{2}\right) \cdot \ldots \cdot\left(1+Y_{N}\right)\right\}
$$

and the procedure would overshoot reality even more. The overshooting effect of autocorrelation further magnifies with the rising intensity of negative autocorrelation.

\section{A Consistent Forecasting Procedure}

The procedure introduced in Section 4 is imperfect, when forecasting for more periods. We then need to find a method which is internally consistent. Internal consistency means that the forecast, if compounded over the relevant horizon $N$, corresponds with the index changes that have occurred (or are expected to occur, given the data) during $N$-year period. The consistency requires the forecasts to differ across forecasting horizons $(N)$. A consistent procedure for a general horizon is the following.

1. Construct the annual yield distribution $D$ from $T$ source observations. (The distribution is likely to be described by a trivial histogram, in which every unique observation occurs with the frequency of $1 / T$ ).

2. Set the initial value of the index to be one, i.e. $I_{0}=1$.

3. Using the annual yield distribution $D$, randomly draw $N$ independent values and apply them to the index. If drawn values are labelled $y_{1}, y_{2}, \ldots, y_{\mathrm{N}}$, we obtain $I_{0} \cdot\left(1+y_{1}\right) \cdot\left(1+y_{2}\right) \cdot \ldots \cdot\left(1+y_{N}\right)=I_{N}$. Thus, we obtain the terminal value of the index after $N$ periods $\left(I_{N}\right)$.

4. Take the $N$-th root of the terminal value of the index after $N$ periods $\left(I_{N}\right)$ and subtract one to obtain compound annual growth rate. In other words, to obtain annual yield $b$, which delivers the above-mentioned terminal index value after $N$ periods, i.e. $b=\left(I_{N}\right)^{1 / N}-1$.

5. Record the annual yield for $N$ periods, the number $b$. We label it $b_{1}$ to explicitly mention it is the first of many simulated final values.

6. Repeat steps 3-5 infinite times or, ideally, pick all permissible combinations of draws. The number of such repetitions we call $j$.

7. Take the arithmetic average of individual annual yields for $N$ periods - numbers $b_{1}, \ldots, b_{j}$. The resulting number is the yield forecast for the $N$-period horizon $\left(R_{N}\right)$ : $R_{N}=\frac{1}{j} \cdot \sum_{i=1}^{j} b_{j}$.

Algebraically written, the forecast is

$$
R_{N}=E\{B\}=E\left\{I_{N}{ }^{1 / N}\right\}-1=E\left\{\left[\left(1+Y_{1}\right) \cdot\left(1+Y_{2}\right) \cdot \ldots \cdot\left(1+Y_{N}\right)\right]^{1 / N}\right\}-1,
$$

where $B$ is a random variable describing possible realizations of number $b$ from step 4 . 
Call this expression the analytical form of the horizon-consistent average for $N$ period forecasting.

Assuming independence of random variables $Y_{i}$ (which also implies zero autocorrelation), it is possible to simplify (15) to:

$$
\begin{aligned}
& E\left\{\left[\left(1+Y_{1}\right) \cdot\left(1+Y_{2}\right) \cdot \ldots \cdot\left(1+Y_{N}\right)\right]^{1 / N}\right\}= \\
& =E\left\{\left(1+Y_{1}\right)^{1 / N} \cdot\left(1+Y_{2}\right)^{1 / N} \cdot \ldots \cdot\left(1+Y_{N}\right)^{1 / N}\right\}= \\
& =E\left\{\left(1+Y_{1}\right)^{1 / N}\right\} \cdot E\left\{\left(1+Y_{2}\right)^{1 / N}\right\} \cdot \ldots \cdot E\left\{\left(1+Y_{N}\right)^{1 / N}\right\}=\left[E\left\{(1+Y)^{1 / N}\right\}\right]^{N}
\end{aligned}
$$

Call the expression (17) the computational form of the horizon-consistent average for $N$ period forecasting. For brevity, the term horizon-consistent will be shortened to consistent in the text below.

$$
R_{N}=\left[E\left\{(1+Y)^{1 / N}\right\}\right]^{N}-1
$$

Although the computational form is less instructive in comparison with the analytical form, it possesses a fundamental advantage in the ease of calculation. The implementation of the analytical form is for longer horizons possible only simulationally. It means in step 8, picking all combinations becomes infeasible and we have to resort to the first-mentioned option of randomized drawing (and, of course, the phrase infinite times has to be commuted to many times, say, 2,000,000). On the contrary, computational form allows calculating exact values of the consistent average for all forecasting horizons in real time and can be implemented even in a spreadsheet.

Notice the innovation of the consistent procedure over the previous inconsistent one. Both approaches firstly simulate terminal values of the index after $N$ periods $\left(I_{N}\right)$, and

- Consistent approach firstly takes the roots and subsequently calculates the mean value from these many roots.

- Inconsistent approach firstly calculates the mean value of the index and subsequently takes the root of it.

In other words, the consistent approach considers the annual yields in every $N$-year scenario, from which then it takes the mean value. The inconsistent approach calculates the key result - the compound annual growth rate - via the mean value of the index after $N$ periods and this by-pass inserts a flaw into the result.

Are the inconsistent values systematically greater or smaller than the consistent ones for some horizons?

The answer lies in Jensen's inequality. ${ }^{8}$ It states that for every convex (or concave) function $g(z)$ holds $g(E\{z\}) \leq E\{g(z)\}$ (or $g(E\{z\}) \geq E\{g(z)\}$ ). Because $g(z)=z^{1 / N}$, where $\mathrm{x}=\left(1+Y_{1}\right) \cdot\left(1+Y_{2}\right) \cdot \ldots \cdot\left(1+Y_{N}\right)$, is concave for $N>1$, Jensen's inequality implies:

$$
\left[E\left\{\left(1+Y_{1}\right) \cdot\left(1+Y_{2}\right) \cdot \ldots \cdot\left(1+Y_{N}\right)\right\}\right]^{1 / N} \geq E\left\{\left[\left(1+Y_{1}\right) \cdot\left(1+Y_{2}\right) \cdot \ldots \cdot\left(1+Y_{N}\right)\right]^{1 / N}\right\}
$$

The inconsistent result (the left-hand side) overshoots the consistent result (the right-hand side) for all forecasting horizons longer than one period (i.e. $N>1$ ). For one-year horizon,

8 The proof is available on http://en.wikipedia.org/wiki/Jensen's_inequality. 
both calculations are equal, as

$$
\left[E\left\{1+Y_{1}\right\}\right]^{1}=E\left\{\left[1+Y_{1}\right]^{1}\right\} .
$$

In other words, in one-year forecasting there is no difference between taking the expected value first and taking its root later, or vice versa, which was the only difference between the procedures. As a result, the arithmetic-average procedure presented in Section 4 is equivalent to the consistent procedure for one-year horizon $(N=1)$.

\section{Biases in Arithmetic and Geometric Averages}

This section examines if arithmetic and geometric averages generally overstate or understate the consistent result presented in Section 5.

\subsection{Systematic error when forecasting with arithmetic average}

Because the procedure presented in Section 4 is based on arithmetic average, the direction of its bias relative to the consistent average has already been discussed. It stems from the following relationships:

$$
\begin{aligned}
& 1+a_{A(T)}=1+E\{Y\}=\left[(E\{1+Y\})^{N}\right]^{1 / N}= \\
& =\left[\left(E\left\{\left(1+Y_{1}\right) \cdot\left(1+Y_{2}\right) \cdot \ldots \cdot\left(1+Y_{N}\right)\right\}\right)\right]^{1 / N} \geq \\
& E\left\{\left[\left(1+Y_{1}\right) \cdot\left(1+Y_{2}\right) \cdot \ldots \cdot\left(1+Y_{N}\right)\right]^{1 / N}\right\}
\end{aligned}
$$

Forecasts using the arithmetic average $\left(a_{A(T)}\right)$ are always consistent for $N=1$ and always overstate the consistent forecast for $N=1$. The degree of overshooting is given by the concavity of the function $g(z)=z^{1 / N}$. Higher $N$ means higher concavity and more serious overshooting. Thus, arithmetic average is most flawed when forecasting for long horizons.

Another determinant of the overshooting magnitude is the dispersion of the past yields in the dataset $\left(x_{i} \text { 's }\right)^{9}$. In the hypothetical case when all past yield are identical, the expression (20) holds as equality. But there is little reason for studying averaging mechanisms when all values are identical.

\subsection{Systematic error when forecasting with geometric average}

Now we investigate the systematic bias of the geometric average, relative to the consistent average. When calculated from $T$ values, geometric average is defined by (28). There are 2 reasons why it deviates from the consistent average:

- Different choice of the forecasting horizon;

- Discrepancy between the geometric average of the entire dataset and the expected value of sample geometric averages.

9 This can be seen from the expression $z$ in the assumptions of Jensen's inequality. $z$ is constant if and only if all the values are identical (and always the same value $y$ is drawn from the distribution). Function $g(z)$ is then defined only in one point, which does not satisfy the convexity/concavity definition and Jensen's inequality cannot be applied. The greater the differences between the $x_{i}$ data, the greater the dispersion of $z$ values. The overshooting of arithmetic is then amplified through the prospective concavity of $g(z)$. 
A formal decomposition to both sources of difference can be made as follows.

$$
a_{K(T)}(N)-a_{G(T)}=\left[a_{K(T)}(N)-a_{K}(T)\right]+\left[a_{K(T)}(T)-a_{G(T)}\right],
$$

where $a_{K(T)}(\cdot)$ is the consistent average for the forecasting horizon in brackets and $a_{G(T)}$ is the geometric average. Subscript $(T)$ indicates they are calculated using a dataset of $T$ observations.

The term in the first square brackets relates to the error caused by the forecasting horizon. The term in the second square brackets relates to the error of association of the geometric average with the expected sample average. Let's investigate both errors in detail.

Forecasting horizon error. It would be nice to consider geometric average to be a special case of a consistent average with a forecasting horizon $N=T$. In other words, to consider that for geometric average $a_{G(T)}$ holds

$$
\begin{aligned}
& 1+a_{G(T)}=\left[\left(1+x_{1}\right) \cdot\left(1+x_{2}\right) \cdot \ldots \cdot\left(1+x_{T}\right)\right]^{1 / T}= \\
& =E\left\{\left[\left(1+Y_{1}\right) \cdot\left(1+Y_{2}\right) \cdot \ldots \cdot\left(1+Y_{T}\right)\right]^{1 / T}\right\}
\end{aligned}
$$

For $T=N$, the expression (22) becomes (after subtracting 1) identical to the expression (15), which describes the consistent result. Thus, for $T=N$ the geometric average equals the consistent one. In other cases, the two are not equal. This is caused by the fact the consistent average diminishes in forecasting horizon.

Why does the consistent average diminish in $N$ ? This can be reformulated to stating that the expected value of the geometric average of values randomly drawn from an identical distribution decreases in the number of values (draws) subsumed in the average. This statement takes the algebraic form of

$$
E\left\{\left[\left(1+Y_{1}\right) \cdot\left(1+Y_{2}\right) \cdot \ldots \cdot\left(1+Y_{N}\right)\right]^{1 / N}\right\}>E\left\{\left[\left(1+Y_{1}\right) \cdot\left(1+Y_{2}\right) \cdot \ldots \cdot\left(1+Y_{N+1}\right)\right]^{1 /(N+1)}\right\}
$$

for all natural $N$. For $N=1$, under the independence assumption the statement is simplified to

$$
E\left\{1+Y_{1}\right\}>E\left\{\left[\left(1+Y_{1}\right) \cdot\left(1+Y_{2}\right)\right]^{1 / 2}\right\}=E\left\{\left(1+Y_{1}\right)^{1 / 2}\right\} \cdot E\left\{\left(1+Y_{1}\right)^{1 / 2}\right\}=\left[E\left\{\left(1+Y_{1}\right)^{1 / 2}\right\}\right]^{2}
$$

Jensen's inequality applied to $z=1+Y_{1}$ and a concave function $g(z)=z^{1 / 2}$ yields

$$
\left[E\left\{\left(1+Y_{1}\right)^{1 / 2}\right\}\right]^{2}<\left[\left(E\left\{1+Y_{1}\right\}\right)^{1 / 2}\right]^{2}=E\left\{1+Y_{1}\right\}
$$

which proves the statement for $N=1$. Now we show the statement holds for a general $N$. Let's rearrange the statement (23) to the form similar to (24):

$$
\left[E\left\{(1+Y)^{1 / N}\right\}\right]^{N}>\left[E\left\{(1+Y)^{1 /(N+1)}\right\}\right]^{N+1} .
$$

Denote $z=(1+Y)^{1 / N .}$ As seen in (27),

$$
\begin{aligned}
& {\left[E\left\{(1+Y)^{1 /(N+1)}\right\}\right]^{N+1}=\left[E\left\{z^{N /(N+1)}\right\}\right]^{N+1}<\left[(E\{z\})^{N /(N+1)}\right]^{N+1}=} \\
& =[E\{z\}]^{N}=\left[E\left\{(1+Y)^{1 / N}\right\}\right]^{N}
\end{aligned}
$$


Jensen's inequality for a concave function $g(z)=z^{N /(N+1)}$ places an upper bound on the righthand side of the inequality (26), and this upper bound is found to be equal to the left-hand side of the inequality (26). This completes the proof.

The recently proven statement means that the consistent average permanently diminishes when the forecasting horizon $N$ increases (until some limit value, as shown later). When using the geometric average $a_{G(T)}$, which is constant and thus independent of forecasting horizon $N$, we implicitly employ a consistent average with a forecasting horizon $T$ for all horizons. As (22) shows, the geometric average is equivalent to the consistent average only for $T=N$. The corollary is that for $N<T$ the geometric average is below the consistent average and for $N>T$ it is above the consistent average.

Error from discrepancy between the geometric average and the expected value. Now we return to (22), which assumed the geometric average of actual data equals to the expected value of geometric average of random draws from their distribution. The statement is, unfortunately, not fully correct. Let us investigate why.

The geometric average $\left(a_{G(T)}\right)$ of all past realized yields $x_{i}$ is defined as:

$$
1+a_{G(T)}=\sqrt[T]{\left(1+x_{1}\right) \cdot\left(1+x_{2}\right) \cdot \ldots \cdot\left(1+x_{T}\right)} .
$$

The expected value of the geometric average of $T$-element independent random selection from the distribution described by $x_{i}^{\prime}$ s is calculated as the arithmetic average form all permissible situations:

$$
E\left\{\left[\left(1+Y_{1}\right) \cdot\left(1+Y_{2}\right) \cdot \ldots \cdot\left(1+Y_{T}\right)\right]^{1 / T}\right\}=\frac{\sum_{i_{1}=1}^{T} \sum_{i_{2}=1}^{T} \ldots \sum_{i_{T}^{\prime}=1}^{T} \sqrt[T]{\left(1+x_{i_{1}}\right) \cdot\left(1+x_{i_{2}}\right) \cdot \ldots \cdot\left(1+x_{i_{T}}\right)}}{T^{T}}
$$

Besides the scenarios in which the random draw is identical to the actual data, the expected value is also affected by scenarios different from the actual data. Due to arithmetic averaging of these unrealized scenarios, the expected value is greater than the geometric average of the actual data (or equal, provided that all yield data are identical).

Let aside the trivial cases in which all yield data in the dataset are the same (including the $T=1$ case) and expressions (28) and (29) are thus obviously equal. We will only consider the cases with (at least two) different numbers. For $T=2$ the statement means:

$$
\begin{aligned}
& 1+a_{G(2)}=\sqrt{\left(1+x_{1}\right) \cdot\left(1+x_{2}\right)}< \\
& <\frac{\sqrt{\left(1+x_{1}\right) \cdot\left(1+x_{1}\right)}+2 \sqrt{\left(1+x_{1}\right) \cdot\left(1+x_{2}\right)}+\sqrt{\left(1+x_{2}\right) \cdot\left(1+x_{2}\right)}}{4}= \\
& =E\left\{\left[\left(1+Y_{1}\right) \cdot\left(1+Y_{2}\right)\right]^{1 / 2}\right\}
\end{aligned}
$$

After rearranging, it can be seen the inequality (30) is equivalent to the statement that 2-element arithmetic average is always greater than 2-element geometric average (again, same numbers case left aside). 
For $T>2$ it can be seen the right-hand side of (29) is the $T$-th power of the arithmetic average of numbers $r_{i}$. After raising the right-hand side of (28) to the power of $T$, one arrives to the $T$-element proof of the inequality between arithmetic and geometric average mentioned in Section 2. This means the expected value is greater than the geometric average of actual data. Because consistent approach is based on expected values, the geometric average will always understate the consistent average.

The geometric average is closer to the consistent one if the dataset is large ( $T$ is large), because the error from associating the geometric average with the expected value is reduced as the samples become more similar to the actual data ${ }^{10}$.

Both errors jointly. We have shown that there exist two sources of bias of the geometric average from the consistent average: first appears when a different forecasting horizon from the number of observations is used (present if $N \neq T$, biasing upwards or downwards) and second stems from the association of the geometric average with the expected value when sampling (always present, biasing downwards). For each $T$, the sum of both biases is removed as $N \rightarrow \infty$, because

$$
\begin{aligned}
& \lim _{N \rightarrow \infty}\left[E\left\{(1+Y)^{1 / N}\right\}\right]^{N}= \\
& =\lim _{N \rightarrow \infty}\left[\frac{\left(1+Y_{1}\right)^{1 / N}+\left(1+Y_{2}\right)^{1 / N}+\ldots+\left(1+Y_{T}\right)^{1 / N}}{T}\right]^{N}=a_{G(T)}
\end{aligned}
$$

It means the consistent average converges to the geometric average with infinite forecasting horizon. Put it differently, the geometric average is consistent only for $N \rightarrow \infty$. In other cases, which include all practical situations, the geometric average always understates the consistent value. The magnitude of error diminishes with increasing forecasting horizon. Application in Section 8 shows the error magnitude diminishes rather quickly.

The choice of forecasting horizon matters. The shorter is the horizon, the greater is the magnitude of understatement when geometric average is used. Obviously, geometric average operates with the largest error when forecasting for one-period $(N=1)$. In the common case of $N<T$, the error caused by an improper forecasting horizon further magnifies the error caused by the association with the expected value (in (21), both square brackets are positive). In the less common case ${ }^{11}$ of $N>T$, the horizon error partially compensates the expected value association error, until full convergence occurs for $N=\infty$ (i.e. $a_{K(T)}(\infty)=a_{G(T)}$ ). Summing up, geometric average always understates the consistent value; most severely, if the forecasting horizon is short. These theoretical conclusions comply with Indro, Lee (1997).

10 The statement is difficult to formulate precisely and prove, because as $T$ increases, is not clear, where do the additional observations come from.

11 In company valuation $N=10$ is typical. The question then arises if 9 and less data points are sufficient. Damodaran (2013b) objects even to using data from developed markets outside the USA, where there are 20-25 annual observations available. His argument goes that standard error of such estimation is too large for practical use. Standard error (if data are independent and identically distributed; both being assumed in this text) equals standard deviation of the data divided by the square root of the number of observations. If the standard deviation is realistically $20 \%, 9$ data points deliver $6 \%$ standard error. SP500 realistic yield estimate being $9 \%$, with $95 \%$ reliability the true yield is between $-2.76 \%$ and $20.76 \%$ (normal quantile used). This is hardly usable. 


\section{A Numerical Illustration}

Let's consider a short numerical example to contribute to better understanding of the consistent averaging procedure and to demonstrate validity of the previous theoretical results.

Assume we have annual index yields only for the 2010-2012 period, thus $T=3$. These yields (Damodaran, 2013) are 14.82\% (2010), 2.07\% (2011) and 15.83\% (2012).

Arithmetic average. The arithmetic average of these yields is:

$$
a_{A(3)}=\frac{0.1482+0.207+0.1583}{3}=0.1091=10.91 \% .
$$

Geometric average. The geometric average of these yields is:

$$
a_{G(3)}=\sqrt[3]{(1+0.1482) \cdot(1+0.207) \cdot(1+0.1583)}-1=0.1072=10.72 \%
$$

Consistent average. We will calculate consistent averages using both the analytical form, which demonstrates the logic of the construction, and the computational form, which demonstrates the relative ease of calculation. We start with the analytical form.

Consistent average differs by the forecasting horizon considered. Here we calculate it for horizons of one, two and three years. For one-year forecasting horizon, i.e. $N=1$, there are only 3 scenarios of index development. We have only 3 source data available and we expect one of them to materialize with equal likelihood. Thus, the consistent average would be

$$
\begin{aligned}
& a_{K(3)}(1)=\frac{1}{3} \cdot(1+0.1482)^{1 / 1}+\frac{1}{3} \cdot(1+0.0207)^{1 / 1}+\frac{1}{3} \cdot(1+0.1583)^{1 / 1}-1= \\
& =0.1091=10.91 \%=a_{A(3)}
\end{aligned}
$$

For two-year forecasting horizon, i.e. $N=2,9$ equally likely index development scenarios might occur (see Table 3).

Table 3 | Two-Year Scenarios for Consistent Average Calculation

\begin{tabular}{|l|c|c|c|c|}
\hline $\begin{array}{c}\text { Scenario } \\
\text { number }\end{array}$ & $\begin{array}{c}\text { First year } \\
\text { yield }\end{array}$ & $\begin{array}{c}\text { Second year } \\
\text { yield }\end{array}$ & $\begin{array}{c}\text { Terminal index value } \\
\text { (initial value = 1) }\end{array}$ & $\begin{array}{c}\text { Compound annual } \\
\text { growth rate }\end{array}$ \\
\hline $\mathbf{1}$ & $2.07 \%$ & $2.07 \%$ & 1.0418 & $2.07 \%$ \\
\hline $\mathbf{2}$ & $2.07 \%$ & $14.82 \%$ & 1.1720 & $8.26 \%$ \\
\hline $\mathbf{3}$ & $2.07 \%$ & $15.83 \%$ & 1.1823 & $8.73 \%$ \\
\hline $\mathbf{4}$ & $14.82 \%$ & $2.07 \%$ & 1.1720 & $8.26 \%$ \\
\hline $\mathbf{5}$ & $14.82 \%$ & $14.82 \%$ & 1.3184 & $14.82 \%$ \\
\hline $\mathbf{6}$ & $14.82 \%$ & $15.83 \%$ & 1.3300 & $15.32 \%$ \\
\hline $\mathbf{7}$ & $15.83 \%$ & $2.07 \%$ & 1.1823 & $8.73 \%$ \\
\hline $\mathbf{8}$ & $15.83 \%$ & $14.82 \%$ & 1.3300 & $15.32 \%$ \\
\hline $\mathbf{9}$ & $15.83 \%$ & $15.83 \%$ & 1.3417 & $15.83 \%$ \\
\hline
\end{tabular}

Source: Damodaran (2013). 
Scenarios are combinations of yields occurring in the first and the second year. Each combination delivers certain terminal value of the index (which is initially set to one) and associated compound annual growth rate. This compound rate equals the geometric average of realized annual growth rates. Consistent average is calculated as the arithmetic average of these 9 compound growth rates:

$$
\begin{aligned}
& a_{K(3)}(2)=\frac{1}{9} \cdot(1.0418)^{1 / 2}+\frac{1}{9} \cdot(1.172)^{1 / 2}+\frac{1}{9} \cdot(1.1823)^{1 / 2}+\frac{1}{9} \cdot(1.172)^{1 / 2}+\frac{1}{9} \cdot(1.3184)^{1 / 2}+ \\
& +\frac{1}{9} \cdot(1.33)^{1 / 2}+\frac{1}{9} \cdot(1.1823)^{1 / 2}+\frac{1}{9} \cdot(1.33)^{1 / 2}+\frac{1}{9} \cdot(1.3417)^{1 / 2}-1= \\
& =\frac{0.0207+0.0826+0.0873+0.0826+0.1482+0.1532+0.0873+0.1532+0.1583}{9}=10.82 \%
\end{aligned}
$$

For three-year forecasting horizon, i.e. $N=3,27$ equally likely index development scenarios

\begin{tabular}{|c|c|c|c|c|c|c|c|c|c|c|c|}
\hline Scenario & $\begin{array}{c}1^{\text {st }} \text { year } \\
\text { yield }\end{array}$ & $\begin{array}{c}2^{\text {nd }} \text { year } \\
\text { yield }\end{array}$ & $\begin{array}{c}3^{\text {rd }} \text { year } \\
\text { yield }\end{array}$ & $\begin{array}{l}\text { Terminal } \\
\text { index } \\
\text { value }\end{array}$ & CAGR & Scenario & $\begin{array}{c}1^{\text {st }} \text { year } \\
\text { yield }\end{array}$ & $\begin{array}{c}2^{\text {nd }} \text { year } \\
\text { yield }\end{array}$ & $\begin{array}{c}3^{\text {rd }} \text { year } \\
\text { yield }\end{array}$ & $\begin{array}{c}\text { Terminal } \\
\text { index } \\
\text { value }\end{array}$ & CAGR \\
\hline 1 & $2.07 \%$ & $2.07 \%$ & $2.07 \%$ & 1.0634 & $2.07 \%$ & 15 & $14.82 \%$ & $14.82 \%$ & $15.83 \%$ & 1.5271 & $15.16 \%$ \\
\hline 2 & $2.07 \%$ & $2.07 \%$ & $14.82 \%$ & 1.1962 & $6.15 \%$ & 16 & $14.82 \%$ & $15.83 \%$ & $2.07 \%$ & 1.3575 & $10.72 \%$ \\
\hline 3 & $2.07 \%$ & $2.07 \%$ & $15.83 \%$ & 1.2067 & $6.46 \%$ & 17 & $14.82 \%$ & $15.83 \%$ & $14.82 \%$ & 1.5271 & $15.16 \%$ \\
\hline 4 & $2.07 \%$ & $14.82 \%$ & $2.07 \%$ & 1.1962 & $6.15 \%$ & 18 & $14.82 \%$ & $15.83 \%$ & $15.83 \%$ & 1.5405 & $15.49 \%$ \\
\hline 5 & $2.07 \%$ & $14.82 \%$ & $14.82 \%$ & 1.3457 & $10.40 \%$ & 19 & $15.83 \%$ & $2.07 \%$ & $2.07 \%$ & 1.2067 & $6.46 \%$ \\
\hline 6 & $2.07 \%$ & $14.82 \%$ & $15.83 \%$ & 1.3575 & $10.72 \%$ & 20 & $15.83 \%$ & $2.07 \%$ & $14.82 \%$ & 1.3575 & $10.72 \%$ \\
\hline 7 & $2.07 \%$ & $15.83 \%$ & $2.07 \%$ & 1.2067 & $6.46 \%$ & 21 & $15.83 \%$ & $2.07 \%$ & $15.83 \%$ & 1.3694 & $11.05 \%$ \\
\hline 8 & $2.07 \%$ & $15.83 \%$ & $14.82 \%$ & 1.3575 & $10.72 \%$ & 22 & $15.83 \%$ & $14.82 \%$ & $2.07 \%$ & 1.3575 & $10.72 \%$ \\
\hline 9 & $2.07 \%$ & $15.83 \%$ & $15.83 \%$ & 1.3694 & $11.05 \%$ & 23 & $15.83 \%$ & $14.82 \%$ & $14.82 \%$ & 1.5271 & $15.16 \%$ \\
\hline 10 & $14.82 \%$ & $2.07 \%$ & $2.07 \%$ & 1.1962 & $6.15 \%$ & 24 & $15.83 \%$ & $14.82 \%$ & $15.83 \%$ & 1.5405 & $15.49 \%$ \\
\hline 11 & $14.82 \%$ & $2.07 \%$ & $14.82 \%$ & 1.3457 & $10.40 \%$ & 25 & $15.83 \%$ & $15.83 \%$ & $2.07 \%$ & 1.3694 & $11.05 \%$ \\
\hline 12 & $14.82 \%$ & $2.07 \%$ & $15.83 \%$ & 1.3575 & $10.72 \%$ & 26 & $15.83 \%$ & $15.83 \%$ & $14.82 \%$ & 1.5405 & $15.49 \%$ \\
\hline 13 & $14.82 \%$ & $14.82 \%$ & $2.07 \%$ & 1.3457 & $10.40 \%$ & 27 & $15.83 \%$ & $15.83 \%$ & $15.83 \%$ & 1.5540 & $15.83 \%$ \\
\hline 14 & $14.82 \%$ & $14.82 \%$ & $14.82 \%$ & 1.5137 & $14.82 \%$ & & & & & & \\
\hline
\end{tabular}
might occur (see Table 4).

Table 4 | Three-Year Scenarios for Consistent Average Calculation

Source: Damodaran (2013). Initial index value is 1. CAGR stands for compound annual growth rate.

The consistent average is then

$$
\begin{aligned}
& a_{K(3)}(3)=\frac{1}{27} \cdot(1.0634)^{1 / 3}+\frac{1}{27} \cdot(1.1962)^{1 / 3}+\ldots+\frac{1}{27} \cdot(1.5405)^{1 / 3}+\frac{1}{9} \cdot(1.1554)^{1 / 3}-1= \\
& =\frac{0.0207+0.0615+\ldots+0.1549+0.1583}{27}=10.79 \%
\end{aligned}
$$


Now we employ the computational form. It has the form

$$
a_{K(T)}(N)=\left[E\left\{(1+Y)^{1 / N}\right\}\right]^{N}
$$

where expectations are always taken from only 3 values, because there are only three observations available. After evaluating at $N$, the respective $N$-period consistent average forecasts are delivered.

$$
\begin{aligned}
& a_{K(3)}(1)=\left[\frac{1}{3} \cdot(1+0.1482)^{1 / 1}+\frac{1}{3} \cdot(1+0.0207)^{1 / 1}+\frac{1}{3} \cdot(1+0.1583)^{1 / 1}\right]^{1}-1=0.1091=10.91 \% \\
& a_{K(3)}(2)=\left[\frac{1}{3} \cdot(1+0.1482)^{1 / 2}+\frac{1}{3} \cdot(1+0.0207)^{1 / 2}+\frac{1}{3} \cdot(1+0.1583)^{1 / 2}\right]^{2}-1=0.1082=10.82 \% \\
& a_{K(3)}(3)=\left[\frac{1}{3} \cdot(1+0,1482)^{1 / 3}+\frac{1}{3} \cdot(1+0,0207)^{1 / 3}+\frac{1}{3} \cdot(1+0,1583)^{1 / 3}\right]^{3}-1=0.1079=10.79 \%
\end{aligned}
$$

This simple numerical exercise illustrates that: (1) arithmetic average is greater than geometric average, (2) for one-period forecasting horizon the consistent average equals the arithmetic one, (3) for more-than-one-period horizon lies the consistent average between the arithmetic and geometric averages, (4) as the horizon increases, the consistent average converges to the geometric average (though full convergence occurs only asymptotically), (5) consistent average can be calculated for arbitrary-long horizon, (6) when the analytical form of the consistent average is used, the number of scenarios needed to consider grows extremely fast with the forecasting horizon, (7) the analytical form and the computational form of the consistent average deliver identical numbers; the computational form only being incomparably faster and easier.

\section{Application to SP500}

In the previous sections it has been shown that except for cases of $N=1$ for the arithmetic average and $N=\infty$ for the geometric average, both averages are systematically biased against the consistent average. Although the theoretical exposition identified the direction of the bias, its magnitudes are generally unclear. Borrowing the valuation example and using the forecasting horizon of $N=10$ recommended by Damodaran (2013b), we cannot decide whether to use the geometric or the arithmetic average. Moreover, we do not know the quantitative effect of shifting to the consistent average instead. In this section, we address these issues numerically for annual SP500 dataset for 1928-2012 period published by Damodaran (2013). This dataset is frequently used by valuation practitioners for future yields estimation; for this reason it was selected for our application.

Three types of average are tested against the consistent average, serving as a benchmark.

- $\quad$ Arithmetic average defined by (32);

- Geometric average defined by (33);

- Weighted average of arithmetic average and geometric average defined by (34), proposed by Indro, Lee (1997). It behaves more like arithmetic average when the forecasting horizon is small relative to the number of observation. Contrarily, 
it behaves more like geometric average when the forecasting horizon approaches the number of observations. ${ }^{12}$

$$
\begin{gathered}
a_{A(T)}=\frac{1}{T} \cdot \sum_{i=1}^{T} x_{i} \\
a_{G(T)}=\sqrt[T]{\left(1+x_{1}\right) \cdot\left(1+x_{2}\right) \cdot \ldots \cdot\left(1+x_{T}\right)}-1 \\
a_{W(T)}(N)=\frac{T-N}{T-1} \cdot a_{A(T)}+\frac{N-1}{T-1} \cdot a_{G(T)}
\end{gathered}
$$

The dataset lists 85 annual yields $(T=85)$. Geometric and arithmetic averages are constant for all forecasting horizons and correspond with Table 1. Weighted and consistent averages are horizon-specific. We will consider horizons up to 85 years. ${ }^{13}$

The result of the analysis is shown in Figure 2. As noted in Section 6, the arithmetic average is consistent for one-year forecasting. For longer forecasting horizons it strongly deviates from the consistent value. Oppositely, the geometric average converges to the consistent average as the forecasting horizon rises. The weighted average linearly connects the two.

Figure 2A demonstrates the geometric average is usually closer to the consistent value than the arithmetic average and is also usually closer than the weighted average. Calculation of their respective deviations (in absolute terms) for each horizon identifies the breaking horizons. Deviations are shown in Figure 2B.

By horizon, the preferable methods are these. For $N=1$, the arithmetic average and the weighted average (being equal by definition) are preferred. For $N=2$, the weighted average is preferred. For longer horizons than 2 years, the geometric average is preferred. Finally for $N=83,84,85$, the weighted average is preferred, as its small upward-shifting arithmetic component partially compensates for the improper association of geometric average with the sample expected value (the second bias from Section 6.2). In general, though, the performance of weighted average is rather disappointing; its weighting scheme poorly follows the hyperbolic course of the consistent average (see Figure 2A) and in most cases, the geometric average is a far better choice (see Figure 2B).

The analysis of SP500 delivers two practical conclusions. First, for the recommended horizons around 10 years, the geometric average is clearly preferable. Even if we substantially deviate from the 10-year forecasting horizon, geometric average will still remain the best choice out of the three generally inconsistent averages.

Second, if we accept the logic of the consistent average, the consistent average is preferred for all horizons. The error arising from using the geometric average instead of the consistent one is 0.2 percentage points, when forecasting for 10 years. It is not much, but still far from being negligible.

12 Notice that for $N=1$ it equals to the arithmetic average (which is consistent in this case) and for $N=T$ it equals to the geometric average (which is near-consistent in this case). Nevertheless, the weighting scheme is rather intuitive than mathematically-founded.

13 The weighted average is not reasonably defined for horizons exceeding the total data span. For other averages, the conclusions for horizons over 85 years can be easily inferred from the analysis presented here. 

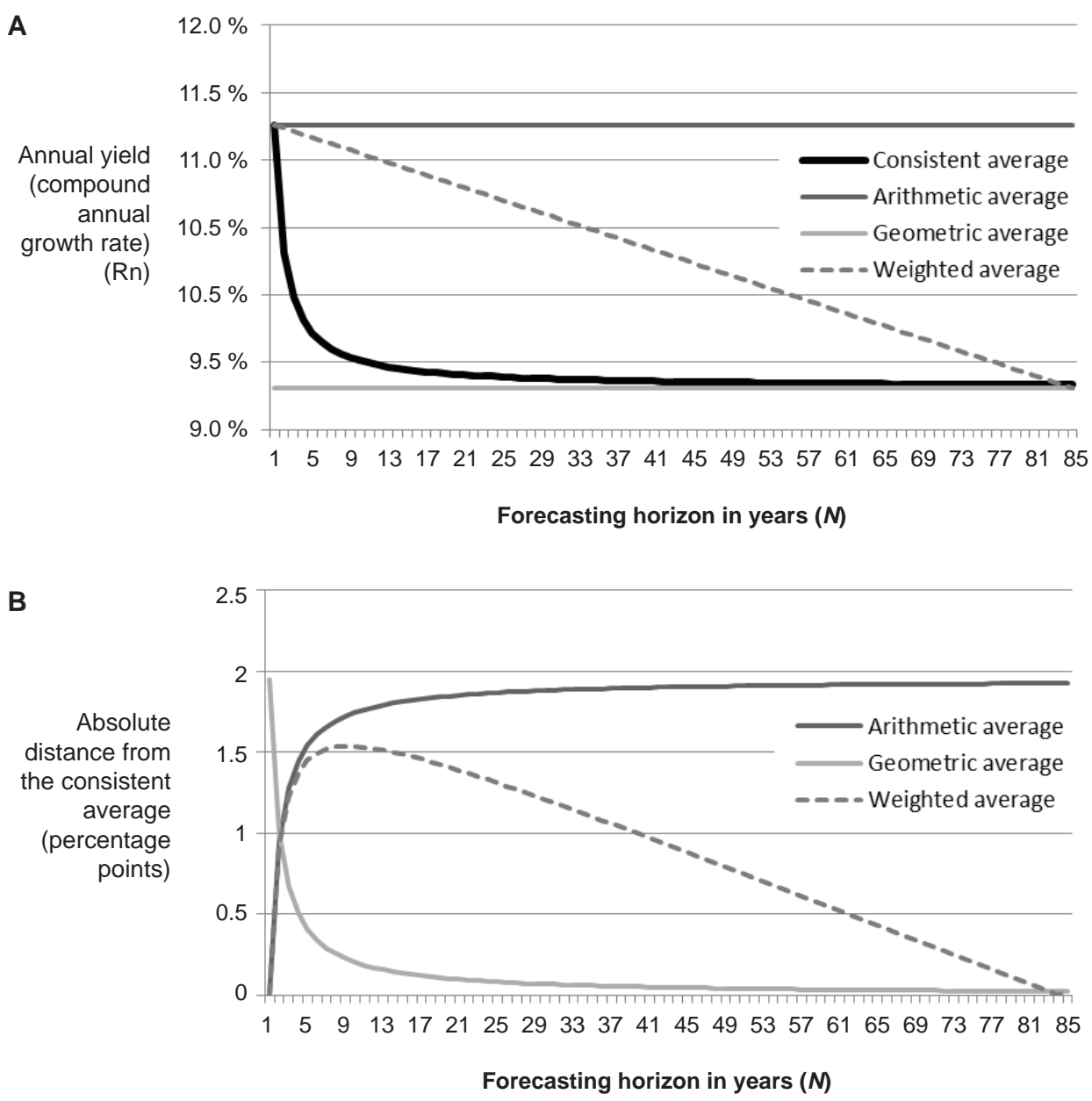

Source: Damodaran (2013).

\section{Conclusion}

The choice of averaging mechanism has a considerable effect on the resulting value of the average past yield or the yield forecast. A consistent solution was proposed here and was called the horizon-consistent average.

Its first step is determining the forecasting horizon for yield forecasts (or the relevant period for past yield measurement). On this horizon, all possible combinations of oneperiod developments that occurred in the dataset are simulated and a geometric average yield is calculated for each scenario. Finally, the horizon-consistent yield is taken as a mean value (arithmetic average) of these yields. It should be mentioned that even with the same dataset of, the horizon-consistent yield differs with different forecasting horizons.

Using this horizon-consistent average as a benchmark, it is possible to discuss biases in arithmetic and geometric averages. For one-year horizon, the arithmetic average equals 
the consistent average. For infinite horizon, the geometric average equals the consistent average. For interlaying horizons, the arithmetic average overstates and the geometric average understates the consistent value.

The application to Ibbotson Associates data for SP500 showed that when estimating the CAPM model and considering the recommended 10-year forecasting horizon to match the average duration of equity and the 10-year risk-free rate (Damodaran, 2008), the geometric average is much closer to the consistent average. For the 1928-2012 annual data, the difference constitutes only 0.2 percentage points, while arithmetic average deviates from the consistent solution by 1.75 percentage points.

In the article, the source data were assumed to be perfect (i.e. non-stochastic). Though the preference of the horizon-consistent average might be questioned when stochastic data are assumed and further investigation of such case is welcome, the horizon-consistent averaging procedure is beneficial in discussing yield measurement and explaining the differences between arithmetic and geometric averages.

\section{References}

Bemerew, D. A. (1999), “Cointegration between Stock Market Indices: The Case of the Slovak and Czech Stock Price Indices." Prague Economic Papers, No. 1.

Damodaran, A. (2013), "Annual Returns on Stock, T. Bonds and T. Bills: 1928 - Current." Stern School of Business. New York University. Available from: http://people.stern.nyu.edu/ adamodar/New_Home_Page/datafile/histretSP.html

Damodaran. A. (2013a), “Estimating Risk Parameters.” Stern School of Business. New York University. Available from: http://people.stern.nyu.edu/adamodar/pdfiles/papers/beta.pdf.

Damodaran. A. (2013b), “Equity Risk Premiums (ERP): Determinants. Estimation and Implications - The 2013 Edition." Stern School of Business. New York University. Available from: http://papers.ssrn.com/sol3/papers.cfm?abstract_id=2238064

Damodaran. A. (2008), "What Is the Riskfree Rate? A Search for the Basic Building Block." Stern School of Business. New York University. Available from: http://people.stern.nyu.edu/ adamodar/pdfiles/papers/riskfreerate.pdf

Dariusz, F. (2013), "Returns and Persistence of Investment Fund Performance in the Czech Republic." Prague Economic Papers, Vol. 22, No. 3, pp. 324-342.

Fama, E. F., French, K. R. (1988), "Permanent and Transitory Components of Stock Prices." Journal of Political Economy, Vol. 96, pp. 246-273.

Indro, D. C., Lee, W. Y. (1997), "Biases in Arithmetic and Geometric Averages as Estimates of Long-Run Expected Returns and Risk Premia." Financial Management, Vol. 26, No. 4, pp. 81-90, http://dx.doi.org/10.2307/3666130

Kavker, A., Festic, M. (2011), “Modelling Stock Exchange Index Returns in Different GDP Growth Regimes." Prague Economic Papers, Vol. 20, No. 1, pp. 3-22.

Mařík, M., et al. (2011), Metody oceňování podniku pro pokročilé: Hlubší pohled na vybrané problémy. Prague: Ekopress.

Trešl, J. (1999), “Prague Stock Exchange: Sectorial Indices Development in 1997." Prague Economic Papers, Vol. 8, No. 1.

Trešl, J., Blatná, D. (2007), “Dynamic Analysis of Selected European Stock Markets." Prague Economic Papers, Vol. 16, No. 4, pp. 291-302.

Veselý, J. (2004), Základy matematické analýzy. První díl. Prague: Matfyzpress. 